\title{
Resolution Enhancement of High Resolution NMR Spectra of Polymers with the Aid of a Computer
}

\author{
Riichirô CHÛJô \\ Department of Polymer Engineering, Tokyo Institute of Technology, Ookayama 2-12-1, Meguro-ku, Tokyo, Japan.
}

Takamune KURODA

Textile Research Institute, Toyobo Co., Ltd., Hon-Katata 1300-1, Otsu, Shiga, Japan.

(Received July 16, 1969)

\begin{abstract}
A method of resolution enhancement of high resolution nuclear magnetic resonance spectra of polymers which are poorly resolved due to large local viscosity, is proposed. This method is a suitable subtraction of the second derivative of the spectra from original ones, and one which can be carried out automatically with the aid of a chart reader and a computer. A moderate value for the proportionality constant to the second derivative is obtained as $K=1-2$. The effect of the separation between representative points at the reading to the computer is also discussed. This procedure is applied to the NMR spectra of poly(methyl propenyl ether).
\end{abstract}

KEY WORDS NMR / Resolution Enhancement / Computer / Second Derivative / Automatic / Poly(Methyl Propenyl Ether) /

Spectroscopic methods have been applied to polymer systems during investigation of their microstructures or their polymerization mechanisms. Such investigations can be said to be quantitative only when the quantitative value of the intensity, position (wave number, frequency, magnetic flux density, etc.) or the shape of peak or valley in the spectra is given with sufficient accuracy. It requires adequate resolution of the spectra. If the discussion is limited to high resolution nuclear magnetic resonance spectroscopy (NMR) of the polymer solution, the resolution is generally poor compared with that in the system of low molecular weight analogue because of the large local viscosity of the former. ${ }^{1}$ The term, "local", is, of course, used here to describe the dimension effective to magnetic dipole-dipole interaction of atomic nuclei. As an example of poor resolution due to large local viscosity, it is known that the more fluid aqueous solution of poly(vinyl alcohol) gives spectra with poorer resolution compared with that of the neat of 2,4-dihydroxypentane as a low molecular weight analogue. ${ }^{2}$ Furthermore, a slight molecular weight dependence on the magnitude of local viscosity has been known in neat or melt of poly(ethylene glycol). ${ }^{3}$ This implies that poor resolution of NMR spectra is inevitable in polymer systems. The need for adequate resolution enhancement of spectra, therefore, may be considered a pre-requisite for carrying out proper quantitative investigations of these systems.

Techniques of resolution enhancement of spectra may be divided into two classes; namely, the improvement in hardware and the introduction of software. The former means obtaining better resolution of spectra by improving magnet homogeneity, the adoption of spectrometers operating on higher frequencies, and other improvements in mechanical or electronic fields. However it has also meant in a practical sense that use of such techniques is limited to the manufacturers of this specialised electronic equipment, due to lack of sufficient skill and knowledge on the part of polymer scientists to make best use of it. Quite apart from this it is not possible for financial reasons to continually bring in the latest equipment as soon as it becomes available. As a result the other technique-improvement by software-has to be introduced. It is suggested that by use of this technique more efficient resolution enhancement can achieved hand-in-hand with increasingly 
effective use of computers and efficiency in the programming. This paper offers one possible method of programming by calculation of the second derivative of spectra, and the method is applied to the NMR spectra of poly(methyl propenyl ether).

\section{PROCEDURE OF COMPUTATION}

It is not true to say that spectra of polymer systems have not been processed before. For example, enhancement of the signal-to-noise ratio of the NMR spectra was successfully carried out for a dilute solution of polyethylene by the accumulation of multiple swept spectra. ${ }^{4}$ A modification of this method was also applied to the NMR spectra of butyl rubber. ${ }^{5}$ These methods, however, cannot be used for the resolution enhancement of poor resolution spectra of polymers. In this case, it is required to distinguish between two or more adjacent signals, not between that of signal and noise.

To do this we suggest superimposition of the second derivative of spectra on to its original spectra. As is well-known, the derivatives, $d^{2 m} f(x) / d x^{2 m}$, of even order of an arbitrary function, $f(x)$, are always positive near the minima or the valleys in the function, while the derivatives are always negative near the maxima or the peaks in the function. Therefore, if

$$
g(\omega)=f(\omega)-\sum_{m-1}^{\infty} C_{m} \frac{d^{2 m} f(\omega)}{d \omega^{2 m}}
$$

is computed for an arbitrary spectrum, $f(\omega)$, the peaks in $g(\omega)$ are higher and sharper than those in $f(\omega)$ and the valleys in $g(\omega)$ are deeper and sharper than those in $f(\omega) .{ }^{6}$ As the simplest form corresponding to this computation,

$$
g(\omega)=f(\omega)-K f^{\prime \prime}(\omega)
$$

is recommended. ${ }^{7}$ The procedure of computation in this paper follows on from this basis. The first matter we must attend to is a reading of $f(\omega)$ which is given as an analogue quantity by the output of a spectrometer. To do this, the abscissa of a chart obtained as an output of a spectrometer is divided into intervals of $0.2 \mathrm{~mm}$ or of its integral times, and the heights of the spectrum at each representative point obtained by the above division is read in a computer, Model JRA-5 (capa- city: $4 \mathrm{k}$ word, 1 word $=16$ bits) with the aid of a chart reader, Model JRA-CR-114. The heights read in are converted into numerical values by the analogue-to-digital converter within the computer.

Noise accompanying the signal is more or less inevitable, and it is greatly amplified by the procedure of differentiation. In order to minimize such effects, the spectrum must be smoothed out before differentiation. The procedure adopted here is to take the average height of the four succeeding representative points, that is,

$$
\bar{f}\left(\omega_{i}\right)=\frac{1}{4} \sum_{j=0}^{3} f\left(\omega_{i-j}\right)
$$

where subscripts for $\omega$ denote the representative points. Taking the average height of a rather small number of points results in the noise remaining, while taking that of too large a number of points tends to cause the characteristics of the spectrum to be lost. The number of points chosen (four) is considered to effect the most suitable compromise from the above point of view. This procedure should hold to reduce the intensities of not only noises but also signals of somewhat less intensities or those eclipsed by stronger ones. The widths of the signals are larger than those of noises in the spectra of polymers. Therefore it isalways possible to carry out a smoothing procedure which affects only noise.

The first derivative of $\bar{f}\left(\omega_{i}\right)$ can be calculated numerically by

$$
\Delta f\left(\omega_{i}\right)=\bar{f}\left(\omega_{i+4}\right)-\bar{f}\left(\omega_{i}\right)
$$

which is again smoothed to

$$
\Delta \bar{f}\left(\omega_{i}\right)=\frac{1}{4} \sum_{j=0}^{3} \Delta f\left(\omega_{i-j}\right)
$$

This leads to the expression

$$
\Delta^{2} f\left(\omega_{i}\right)=\Delta \bar{f}\left(\omega_{i+3}\right)-\Delta \bar{f}\left(\omega_{i-1}\right)
$$

for the smoothed second derivative of $f\left(\omega_{i}\right)$. Then, as the expression for the enhanced spectrum of $f\left(\omega_{i}\right)$, we have

$$
\begin{aligned}
g\left(\omega_{i}\right) & =f\left(\omega_{i}\right)-K \Delta^{2} f\left(\omega_{i}\right) \\
& =\frac{K}{16}\left\{-f\left(\omega_{i-7}\right)-2 f\left(\omega_{i-6}\right)-3 f\left(\omega_{i-5}\right)\right. \\
& -4 f\left(\omega_{i-4}\right)-f\left(\omega_{i-3}\right)+2 f\left(\omega_{i-2}\right)+5 f\left(\omega_{i-1}\right) \\
& +(16 / K+8) f\left(\omega_{i}\right)+5 f\left(\omega_{i+1}\right)+2 f\left(\omega_{i+2}\right)
\end{aligned}
$$




\section{R. CHÛJô and T. KuRodA}

$$
\begin{aligned}
& -f\left(\omega_{i+3}\right)-4 f\left(\omega_{i+4}\right)-3 f\left(\omega_{i+5}\right) \\
& \left.-2 f\left(\omega_{i+6}\right)-f\left(\omega_{i+7}\right)\right\}
\end{aligned}
$$

where $K$ is a positive definite constant. Eq. 7 is, of course, calculated by the computer.

The definite integral of $-K f^{\prime \prime}(\omega)$ from one valley to the next vanishes. Thus the intensity of every peak in a spectrum is kept constant by this procedure of resolution enhancement. This enables us to calculate the intensity of every peak from that of corresponding enhanced peak. This calculation is also carried out by the computer.

The proportionality constant $K$ can be estimated theoretically, if the shape of the spectrum is known. $^{6}$ However, the procedure adopted in the paper is to obtain enhanced spectra corresponding to some appropriate value of $K$, and to decide the most appropriate value of $K$ by the comparison of these outputs visually by the researcher. A similar procedure is also carried out to decide the interval between representative points, which is hereafter denoted by $\Delta$. The shape of the NMR spectrum of polymers is not a simple Gaussian or Lorentzian one, because of the distribution of correlation times. This is one reason why we do not use the theoretical value of $K$. The other reason stems from the fact that higher derivatives of the spectrum appearing in Eq. 1 cannot be ascertained by our procedure. The theoretical value of $K$ has a definite meaning only when the higher derivatives are taken into account at the same time.

\section{AN EXAMPLE OF COMPUTATION}

The procedure described in the previous section was applied to the $\beta-\mathrm{CH}_{3}$ peak in the high resolution NMR spectrum of poly(methyl propenyl ether). The conditions of polymerization and that of the measurement of the NMR spectrum are described elsewhere. ${ }^{8}$ The $\alpha-\mathrm{OCH}_{3}$ peak in the NMR spectrum of the polymer was split into three parts due to the chemical shift corresponding to its three dyad ditacticities. On the other hand, the $\beta-\mathrm{CH}_{3}$ peak decoupled from its $\beta-\mathrm{CH}$ proton appeared to be split into two despite the possibility of a threefold splitting corresponding to the three dyad ditacticities. In discussing the polymerization mechanism of the polymer, it is important to be able to explain the reason for the lack of the third peak, i.e., whether its disappearance is due to a particular choice of configuration in the propagation step, or to a poor resolution of the spectrum. It is for this reason that the $\beta-\mathrm{CH}_{3}$ peak of the polymer is chosen as an example.

Figure 1(a) is the $\beta-\mathrm{CH}_{3}$ peak decoupled from its $\beta-\mathrm{CH}$ proton in the high resolution NMR spectrum of poly(methyl propenyl ether) polymerized with $\mathrm{BF}_{3} \cdot \mathrm{O}\left(\mathrm{C}_{2} \mathrm{H}_{5}\right)_{2}$ in toluene at $-78^{\circ} \mathrm{C}$. This is not the original spectrum, but the output from the computer without any processing such as smoothing or resolution enhancement. For practical purposes, therefore, it can be considered as the original. A line equal to $1 \mathrm{~cm}$ in the original spectrum is shown in the figure to enable comparison of the separation, $\Delta$, between representative points to be made. Figures 1 (b)-(f) show the change of resolution in enhanced spectra due to the change of the proportionality constant, $K$, under the (a)

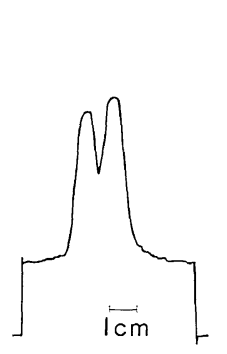

(d)

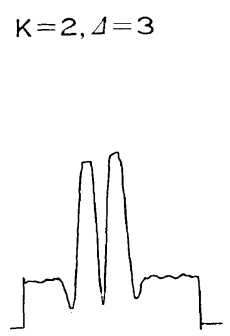

(e)

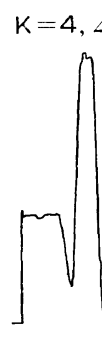

(b)

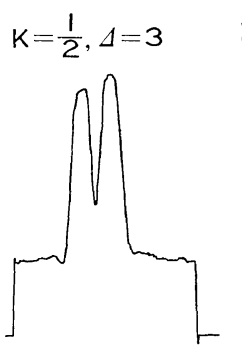

(c)

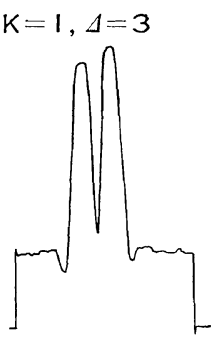

(f)
Figure 1. Changes in the shapes of enhanced spectra of $\beta-\mathrm{CH}_{3}$ protons decoupled from $\beta-\mathrm{CH}$ protons in NMR spectrum of poly(methyl propenyl ether) with the proportionality constant, $K$, to second derivative under the condition of the constant separation, $\Delta=3$, between two adjacent representative points. (a): Not enhanced, (b): $K=1 / 2$, (c): $K=1$, (d): $K=2$, (e): $K=4$, (f): $K=8$. 
(a)

(b)

(c)
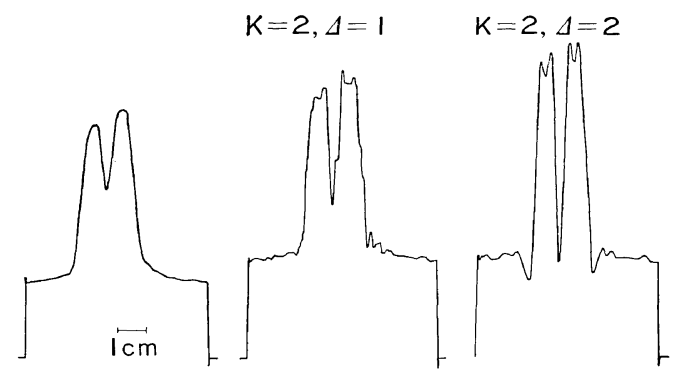

(d)

(e) (a)

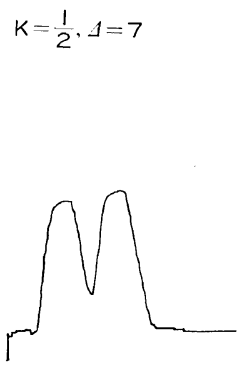

(b)

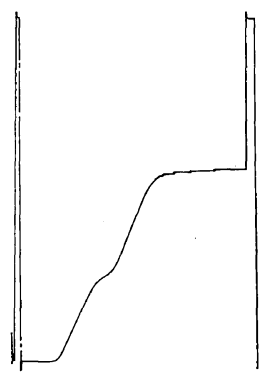

Figure 3. The enhanced spectrum of $\beta-\mathrm{CH}_{3}$ protons decoupled from $\beta-\mathrm{CH}$ protons in NMR spectrum of poly(methyl propenyl ether) with $K=1 / 2, \Delta=7$ (a) and its integrated spectrum (b).

similar to large $K$ values which amplify the effects of the second derivative. Thus if a larger $\Delta$ value is used, the $K$ value must be decreased despite the conclusion stated in the preceding paragraph.

When one half and seven are chosen for the

Figure 2. Changes in the shapes of enhanced spectra of $\beta-\mathrm{CH}_{3}$ protons decoupled from $\beta-\mathrm{CH}$ protons in NMR spectrum of poly(methyl propenyl ether) with the separation, $\Delta$, between two adjacent representative points under the condition of the constant proportionality constant, $K=2$, to second derivative. (a): Not enhanced, (b): $\Delta=1$, (c): $\Delta=2$, (d) $: \Delta=3$, (e): $\Delta=4$, (f): $\Delta=5,(\mathrm{~g}): \Delta=6$.

condition of constant separation, $\Delta=3$ (representative points are chosen at every $0.6 \mathrm{~mm}$ intervals). It can be seen that smaller $K$ values are insufficient for enhancement, while larger $K$ values are inadequate because of larger estimation of the second derivatives compared with the original curves. It can be concluded that moderate $K$ values $(K=1-2)$ give better resolution in this case.

The choice of the separation, $A$, is believed to affect the procedure of smoothing. Figure 2(a) is identical with Figure 1(a), and Figure 2(b)-(g) are enhanced spectra of Figure 2(a) with various values of $\Delta$ under the condition of constant $K$. Smaller values of $\Delta$ are insufficient to prevent the effect of noise which causes the apparent splitting of the peaks as seen in Figure 2(c). The larger the value of $\Delta$, the better the enhancement accomplished within the range of $\Delta$ from one $(0.2 \mathrm{~mm})$ to six $(1.2 \mathrm{~mm})$. It can be concluded in this case that rather large $\Delta$ values cause effects values of $K$ and $\Delta$, respectively, a result as depicted in Figure 3(a) is obtained. Furthermore, the integration of the curve in the figure is shown in Figure 3(b). From these two figures, it can be seen that the third peak is not eclipsed in the range of the other two peaks, so that the third peak essentially disappears in the polymer. This conclusion provides support for the polymerization mechanism described elsewhere. ${ }^{8}$ In addition, the relative intensities of the two peaks, evaluated as 0.44 and 0.56 from Figure 3(b), also lend support to the calculation of relative intensities without resolution enhancement. ${ }^{8}$

\section{CONCLUSION}

A means of resolution enhancement of a spectrum can be successfully achieved by the subtraction of its second derivative, and this can be carried out automatically with the aid of a chart reader and computer. This procedure was applied to the $\beta-\mathrm{CH}_{3}$ peak in the high resolution NMR spectrum of poly(methyl propenyl ether), and the results support those obtained without resolution enhancement.

\section{REFERENCES}

1. F. A. Bovey, G. V. D. Tiers, and G. Fillipovich, 


\section{R. Chû̉ô and T. KurodA}

J. Polym. Sci., 38, 73 (1959).

2. S. Satoh, R. Chûjô, T. Ozeki, and E. Nagai, Repts. Prog. Polym. Phys. Japan, 5, 251 (1962).

3. R. Chûjô, K. Aoki, S. Satoh, and E. Nagai, J. Polym. Sci. Part B, 1, 501 (1963).

4. Y. Kato and A. Nishioka, Bull. Chem. Soc. Japan, 39, 1426 (1966).

5. H. Y. Chen and J. E. Field, J. Polym. Sci., Part
B, 5, 501 (1967).

6. L. C. Allen, H. M. Gladney, and S. H. Glarum, J. Chem. Phys., 40, 3135 (1964).

7. R. R. Ernst, R. Freeman, B. Gestblom, and T. R. Lusenbrink, Mol. Phys., 13, 283 (1967).

8. Y. Ohsumi, T. Higashimura, R. Chûjô, T.Kuroda, and S. Okamura, J. Polym. Sci., Part A-1, 5, 3009 (1967). 